\title{
ACTIVE REFLECTORS FOR INTERFEROMETRIC SAR DEFORMATION MEASUREMENT
}

\author{
Y. Wassie ${ }^{1, *}$, M. Crosetto ${ }^{1}$, G. Luzi $^{1}$, O. Monserrat ${ }^{1}$, A. Barra ${ }^{1}$, R. Palamá ${ }^{1}$, M. Cuevas-González ${ }^{1}$, \\ S.M. Mirmazloumi ${ }^{1}$, P. Espín-López ${ }^{1}$, B. Crippa ${ }^{2}$ \\ ${ }^{1}$ Centre Tecnològic de Telecomunicacions de Catalunya (CTTC/CERCA), Division of Geomatics, Av. Gauss, 7 E-08860 \\ Castelldefels (Spain) - (ywassie, mcrosetto, gluzi, omonserrat, abarra, rpalama, mcuevas, mmirmazloumi, pespin)@cttc.cat \\ ${ }^{2}$ Department of Earth Sciences, Section of Geophysics, University of Milan, Via Cicognara 7, I-20129, Milan, Italy - \\ bruno.crippa@unimi.it
}

Commission III, WG III/3

KEY WORDS: Deformation, monitoring, SAR, active reflectors

\begin{abstract}
:
This paper is focused on the design, implementation and testing of an active reflector, to be used to support deformation monitoring studies based on Synthetic Aperture Radar interferometry. The device is designed to work in C-band with Sentinel-1 data, operating at $5.405 \mathrm{GHz}+50 \mathrm{MHz}$. A brief description of the active reflector is provided. It consists of two antennas and an amplifying section. The active reflector has been tested in different experiments. In this paper, we describe the experiment carried out in the Parc Mediterrani de la Tecnologia (Castelldefels, Barcelona). The result shows a strong correlation with temperature. A calibration test was carried out to experimentally derive a calibration curve to correct the effect of temperature on phase stability.
\end{abstract}

\section{INTRODUCTION}

Differential SAR Interferometry (DInSAR) relies on the presence of good targets in the imaged area, which remain coherent between two acquisitions (Bamler and Hartl, 1998; Rosen et al., 2000; Hanssen, 2001). In the Persistent Scatterer Interferometry (PSI) technique, which makes use of large stacks of SAR images (Crosetto et al., 2016), the coherence of the targets needs to be maintained over the entire observation period (from the first to the last image of the stack). The presence of good targets is usually guaranteed in urban, periurban and industrial areas. By contrast, in vegetated and forested areas, such good targets are rare or often not existing. If one needs to monitor such areas using DInSAR or PSI, the installation of artificial reflectors is necessary. The most used solution is the installation of Passive Corner Reflectors (PCR), e.g. see an example in Crosetto et al. (2013).

A PCR is a target with a simple geometrical shape, designed to perform a high radar reflectivity, i.e. a strong response to the SAR microwaves. PCRs are usually made of metallic elements, with a size that is considerably larger than the radar wavelength, and a shape that maximizes the energy backscattered to the radar. Different types of PCRs have been proposed; the most used is the Triangular Trihedral geometry, e.g. see Garthwaite (2017). Especially in C-band, the PCRs have considerable dimensions, which make them heavy and difficult to deploy, and can have problems in areas characterized by strong wind.

An alternative to PCRs is given by the Active Reflectors (ARs). They are usually smaller, lighter and easy to deploy. An AR basically receives the SAR signal and re-transmits an amplified version of the same. In this way, it provides a strong radar response, which is seen as a bright pixel in the corresponding SAR image. If the device works properly, the response remains stable over time.
ARs require a power source, which is typically provided by a battery or a solar panel, which can be seen as a drawback of the ARs. At the beginning, ARs, which are also known as transponders, were very expensive devices used to radiometrically calibrate the imagery of the different SAR missions. In the last decade, research centres and companies have started to develop ARs, e.g. see Mahapatra et al. (2013) and Metasensing (2020). However, to the opinion of the authors, the high cost of these devices hinders their wider use.

This document reports the design and the tests of ARs operating at $5.405 \mathrm{GHz} \pm 50 \mathrm{MHz}$. These ARs are designed to work with the Copernicus Sentinel-1A and Sentinel-1B sensors. The target of the development of the ARs was a low-cost system, with low power consumption, and portable with simple installation procedures.

\section{DESIGN OF AN ACTIVE REFLECTOR}

This section briefly describes the AR developed and implemented at CTTC. A more technical description is provided in Luzi et al. (2021). The designed AR consists of two antennas and an amplifying section, powered by a battery or any other available power source. A scheme of the device is illustrated in Figure 1.

A key parameter for the design is the Radar Cross Section (RCS), see Skolnik (1990). This depends on four terms: (i) the amplifying section gain; (ii) the receiving antenna gain; (iii) the transmitting antenna gain; (iv) the square of the wavelength (Freeman et al., 1990). Considering the values published for the PCRs, we fixed as a requirement a RCS greater than $40 \mathrm{dBsm}$. The gain of the two antennas was assumed to be greater than 15 dB. A specific antenna was designed. A 4 by 4 linear patch array was designed to provide a gain above $15 \mathrm{~dB}$, reducing the necessary gain of the amplifying section.

\footnotetext{
* Corresponding author
} 


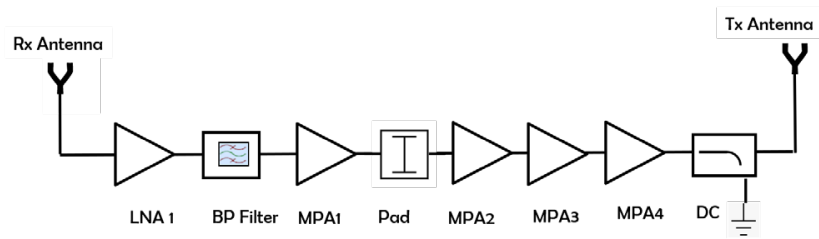

Figure 1. Scheme of the developed AR.

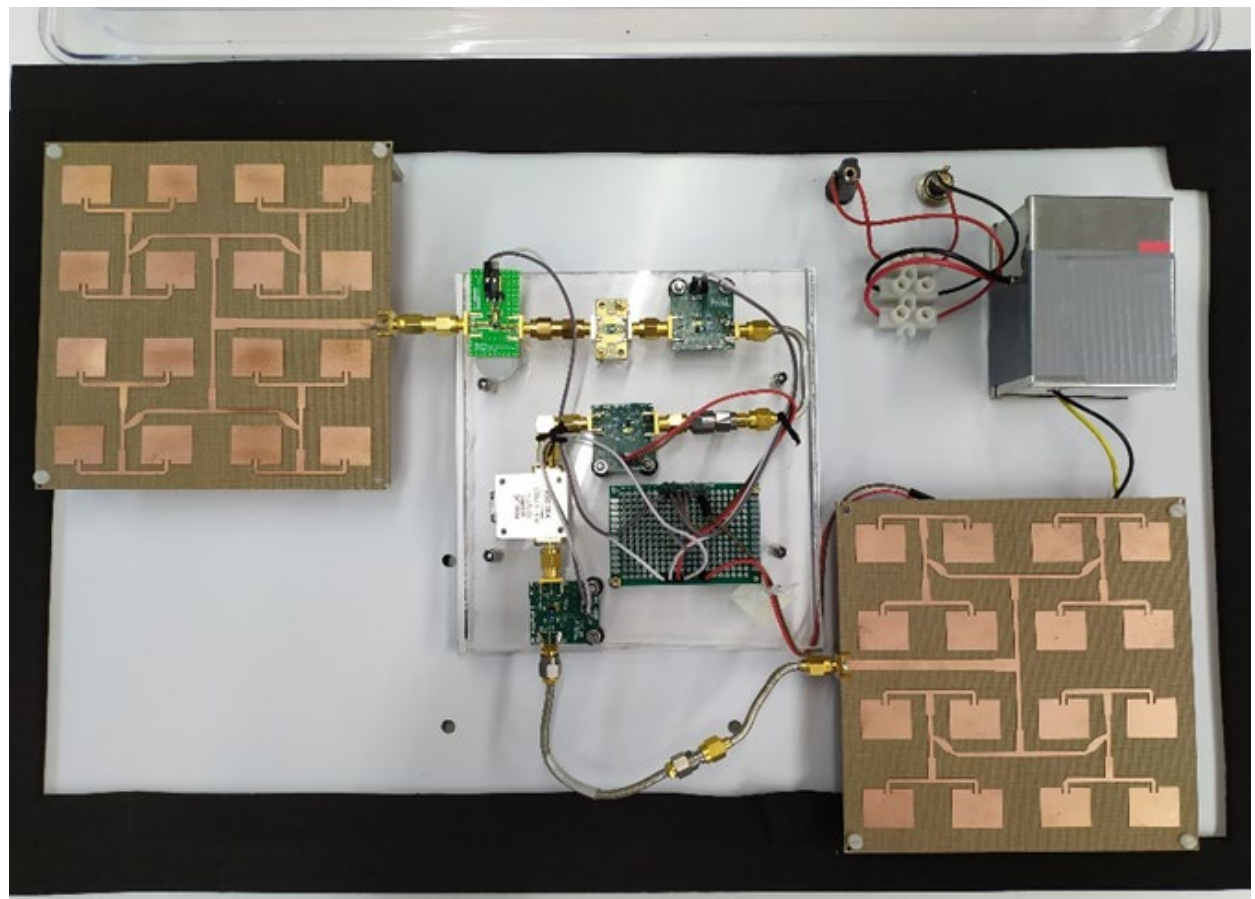

Figure 2. Antennas and amplifying section of one of the AR prototypes.

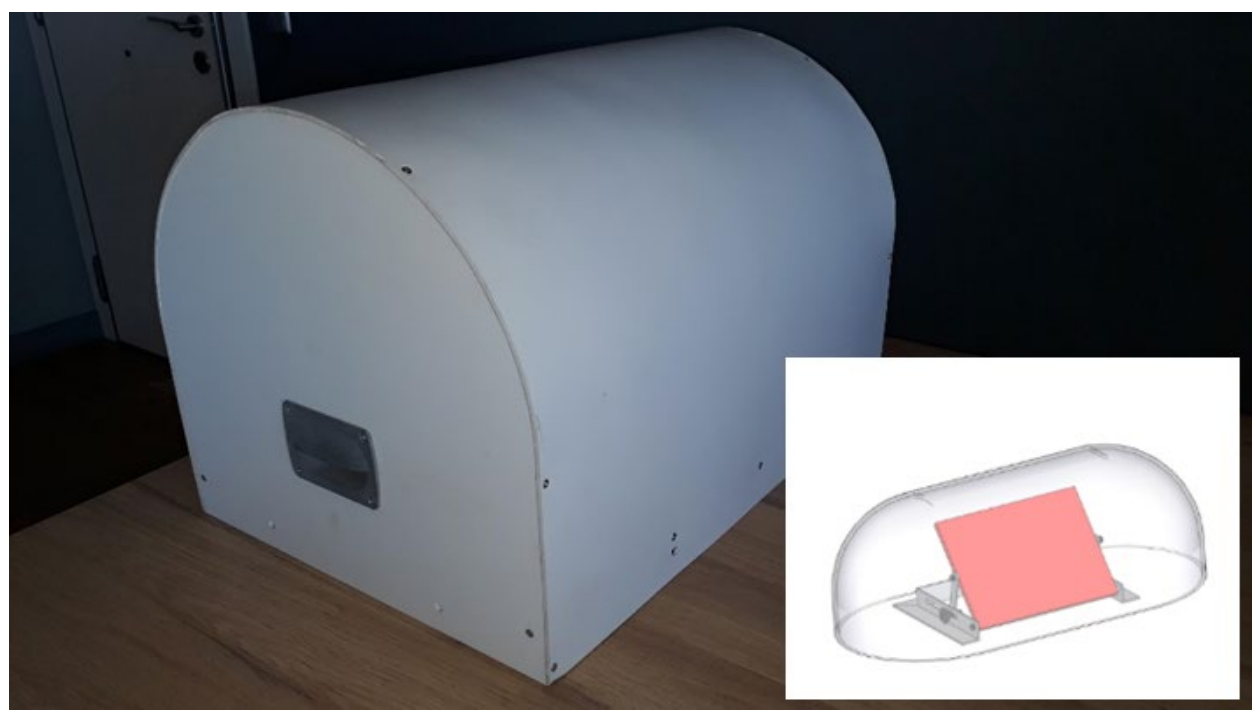

Figure 3. Picture of the external cover of the AR with a scheme of the mechanical frame.

The amplifying section was built using off-the-shelf components. An accurate market analysis was performed to select the single components, considering the performance, cost and component availability. In this design, the gain of the amplifying section was supposed to be not lower than $42 \mathrm{~dB}$.
The amplifying section needs to include a band-pass filter to reduce interference from external sources, e.g. wireless or mobile networks. Figure 2 shows the antennas and amplifying section of one of the AR prototypes, while Figure 3 shows the external cover. 


\section{EXPERIMENTAL RESULTS}

We describe in this paper an experiment carried out in the Parc Mediterrani de la Tecnologia, PMT (Castelldefels, Barcelona). Figure 4 shows the AR used in this experiment: one may notice that the external cover of this prototype differs from the one shown in Figure 3: this was an older prototype version. The experiment included the following steps.

At the beginning we installed a PCR, and an AR. In the analysis, a natural reflector was also considered. The SAR image collection lasted almost one year: from 24 October 2019 to 19 September 2020. In this period, 56 Sentinel-1 images were acquired. The images were co-registered, and a set of interferograms was generated, using pairs of temporally consecutive images. A total of 55 interferograms were analysed. The phase unwrapping of such interferograms was then performed. The interferometric phases were referred to the same reference pixel. The phases were then integrated, generating the temporally ordered phases. Finally, the phases were transformed in displacements, expressed in [mm], see Figure 6.

Figure 5 shows a quick look of an amplitude image that corresponds to the area of interest. The image was displayed using the Sentinel Hub browser of ESA. In this geocoded Sentinel-1 SAR amplitude the AR, the PCR and the natural reflector are clearly visible.

The displacement time series of the AR is shown in Figure 6. This figure includes two time series. The green line shows the estimated deformation time series, whose values are indicated in the left-side axis (the right axis indicates the temperatures). Between 24/10/19 and 24/4/20 the time series is basically stable, with a dispersion of $\pm 2 \mathrm{~mm}$. A drop can be noted between $24 / 4 / 20$ and 24/5/20, while afterwards the time series is stable again. This time series concerns a location that is expected to be stable during the observed period. It is worth to note that the deformation is strongly correlated with temperature (blue line in Figure 6).

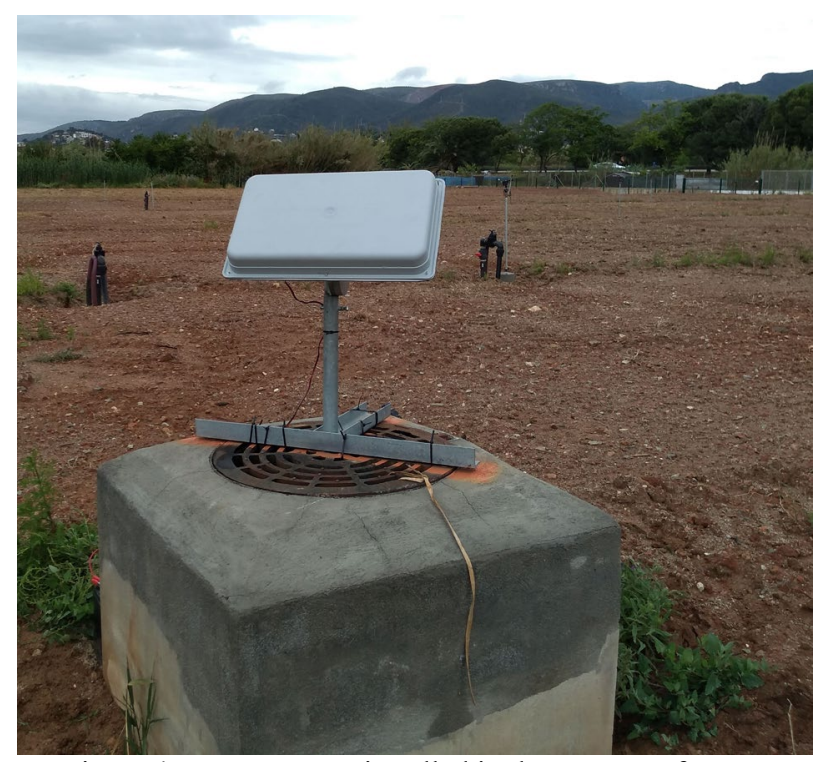

Figure 4. AR prototype installed in the campus of CTTC.

\section{CHARACTERIZATION AND CALIBRATION}

Figure 6 clearly shows a dependency of the "virtual displacements" to temperature. This is confirmed in Figure 7, the deformation and the temperature show a linear correlation, with $\mathrm{R}^{2}$ of 0.84 .

A characterization of the above behaviour was performed, with the aim of finding a calibration procedure for the AR measurements, by measuring the thermal stability of the AR at a short range. In this case, we used an input signal generated by a vector network analyser. The temperature variations were measured using a sensor installed inside the AR. The test lasted 3 days and 18 hours. The sampling interval was 30 minutes, and the temperature range was: $12.5-31^{\circ} \mathrm{C}$.

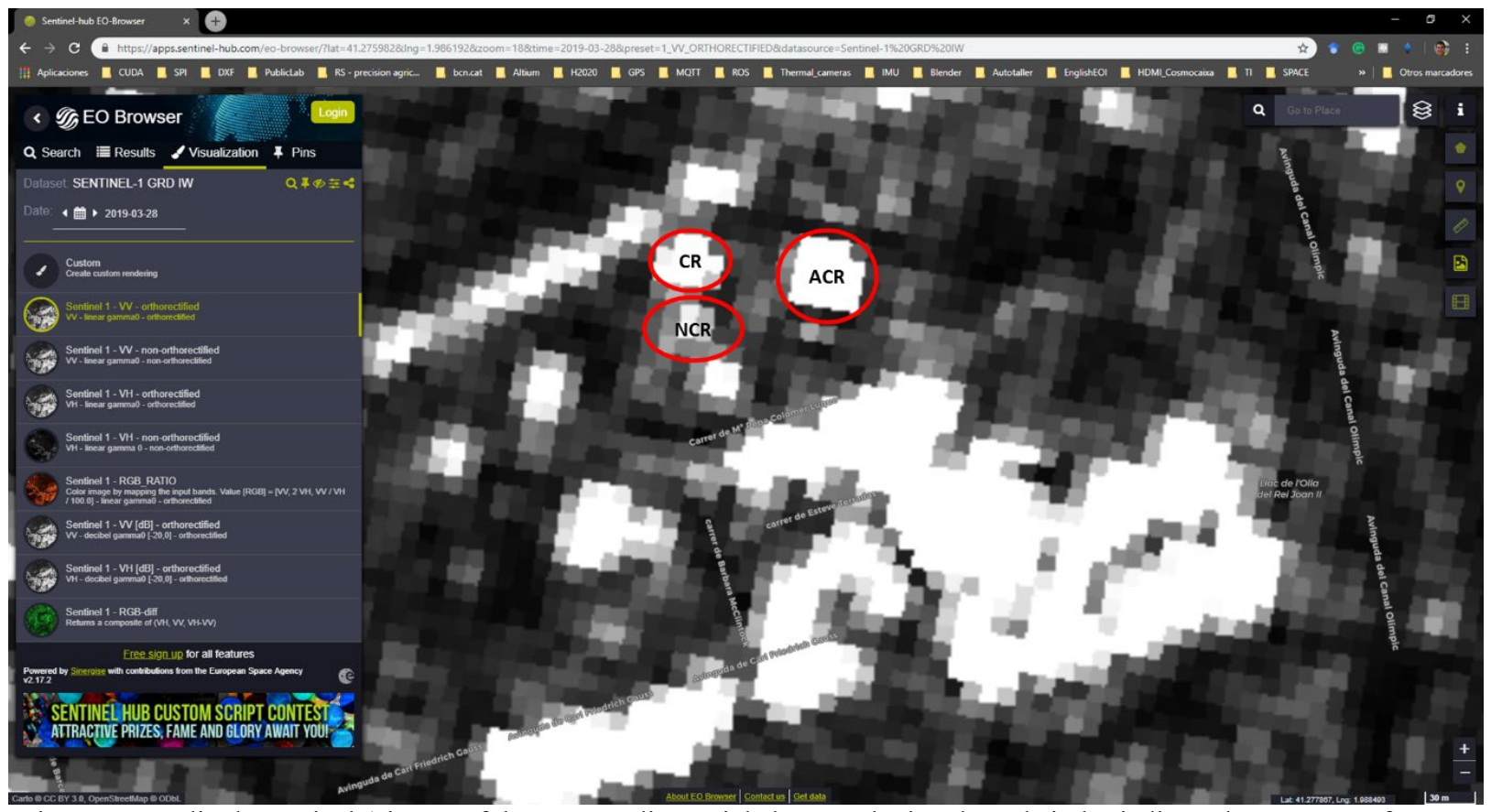

Figure 5. Amplitude Sentinel-1 image of the Parc Mediterrani de la Tecnologia. The red circles indicate the response of an AR (ACR), a passive reflector (CR) and a natural reflector (NCR). 


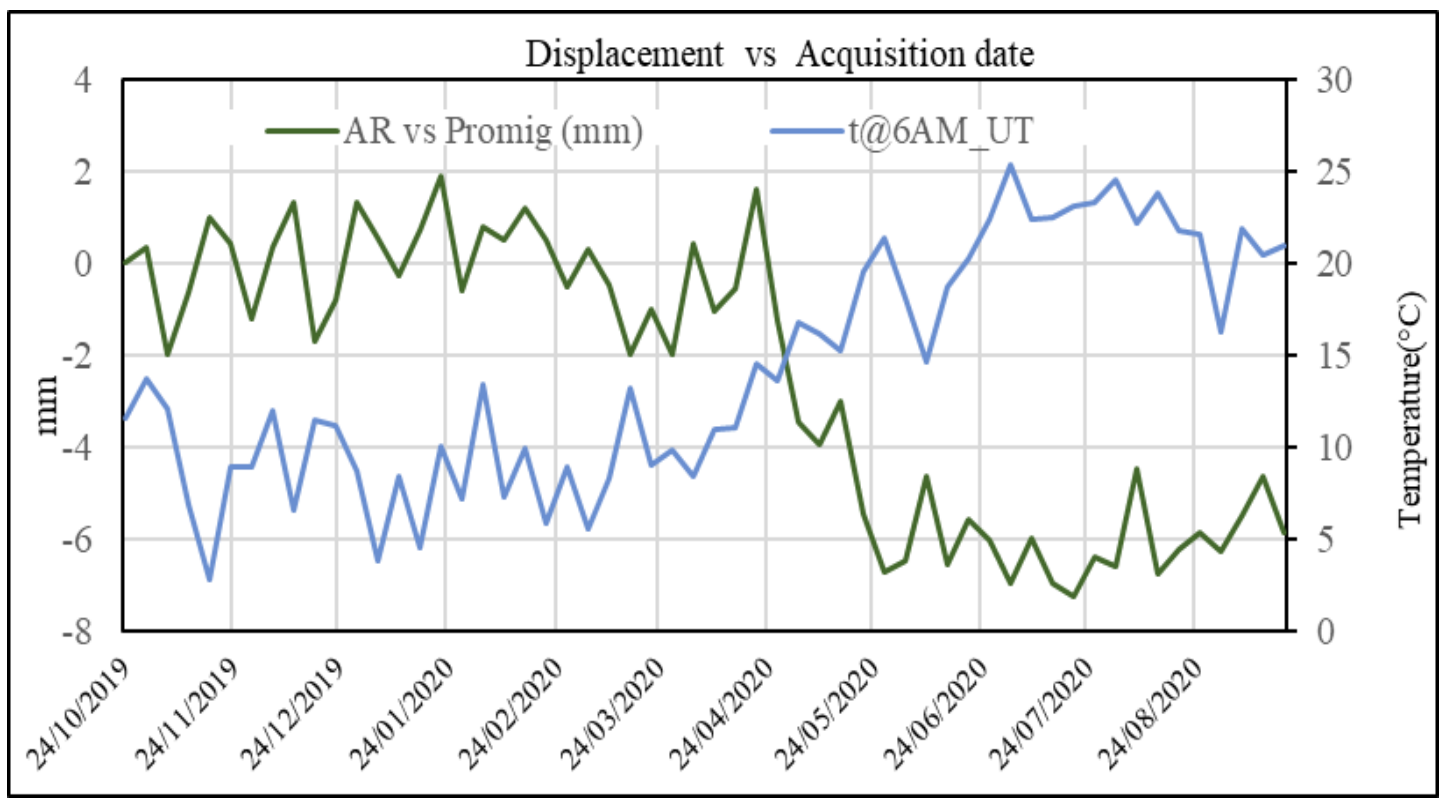

Figure 6. Deformation (green line) and temperature (blue line) time series.

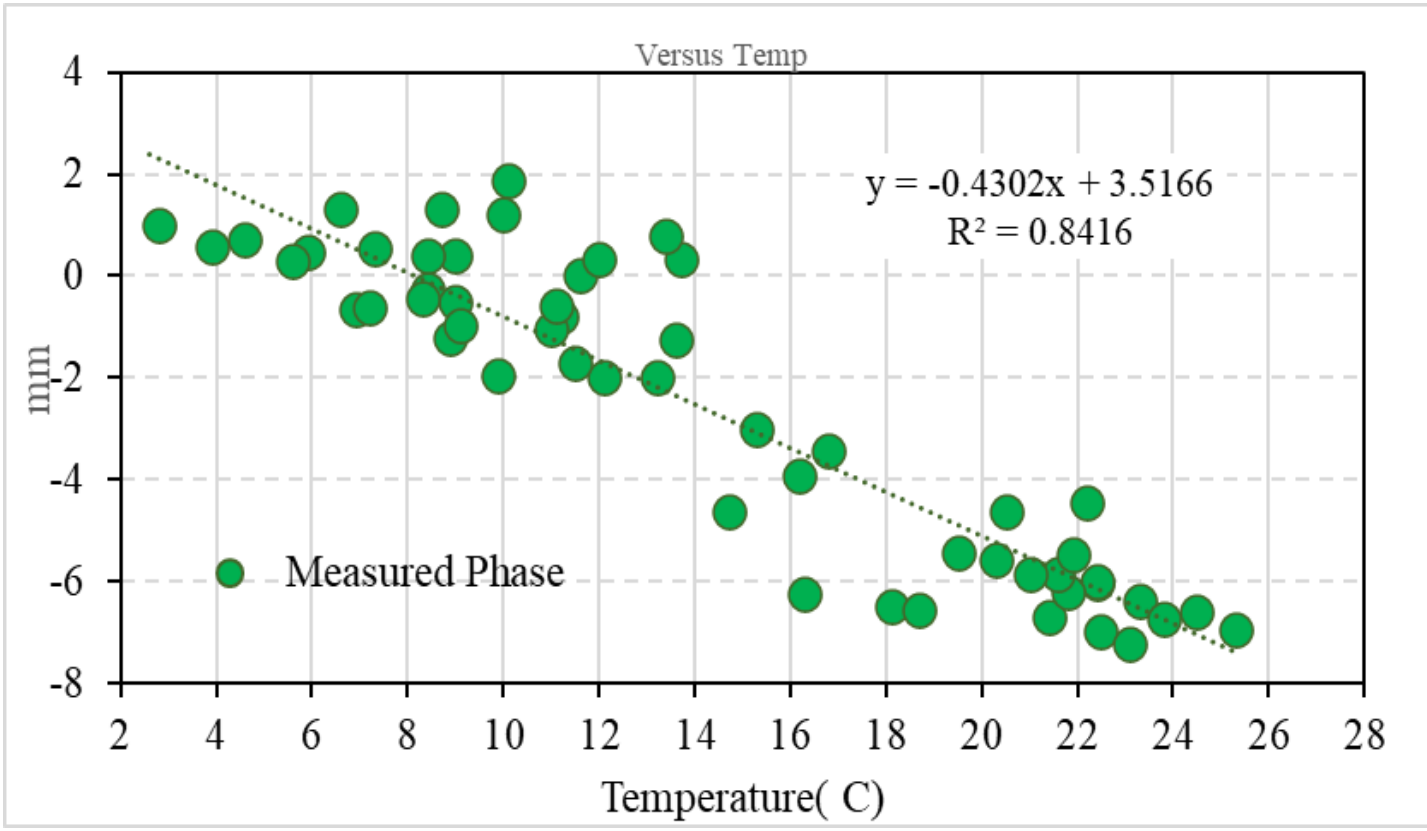

Figure 7. Plot of the deformation, vertical axis in [mm], versus temperature.

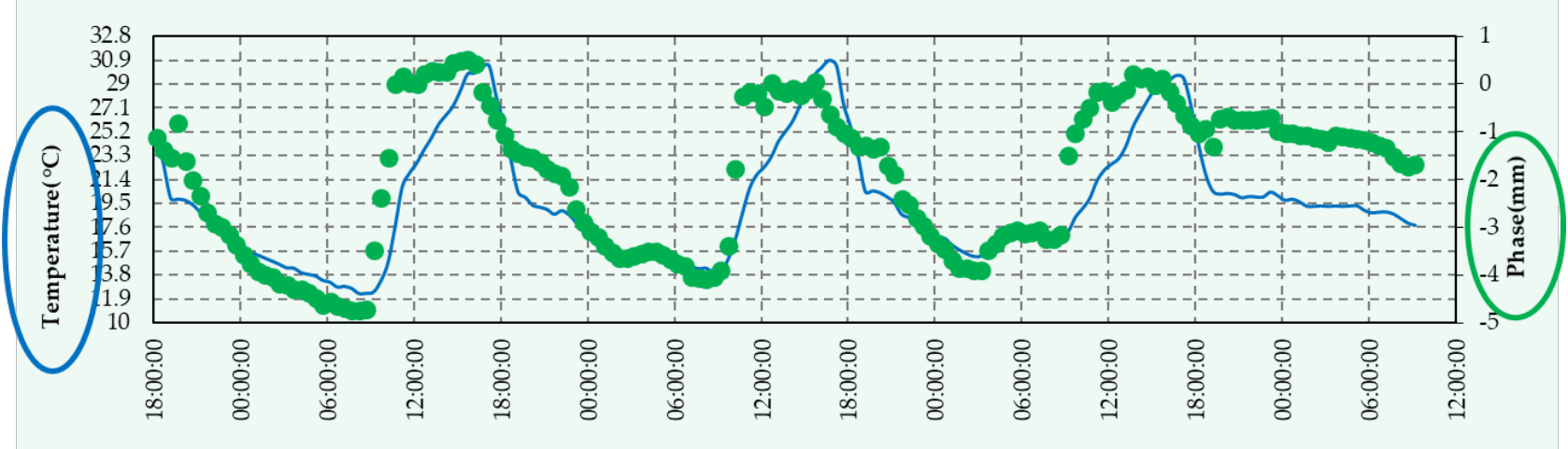

Figure 8. Displacement (phase) and temperature of the AR measured during the calibration. The blue line represents temperature (see the left y-axis), while the green dots indicate displacement values (see the right y-axis). Modified from Luzi et al. (2021). 


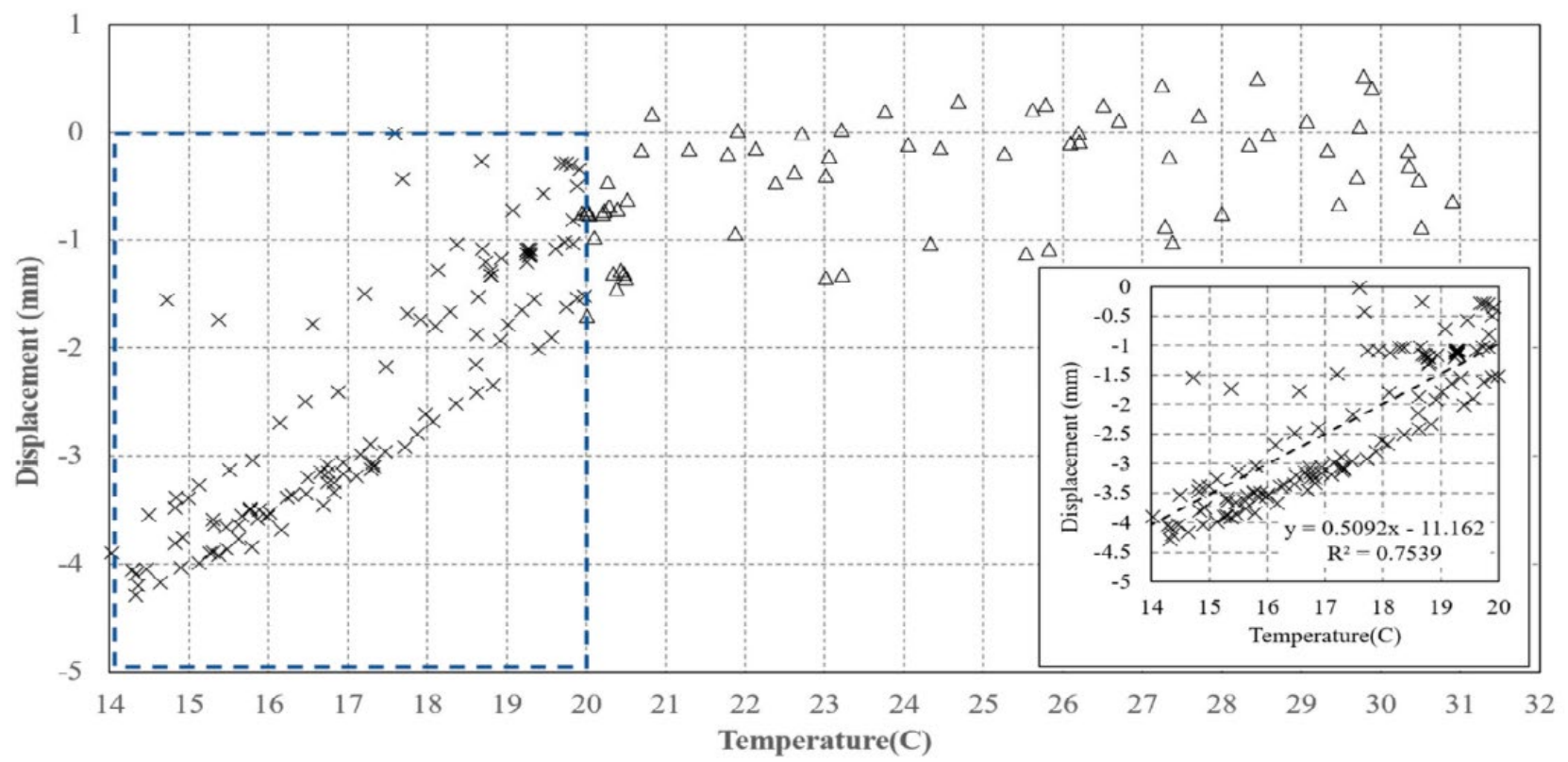

Figure 9. Displacement of the AR versus temperature measured during the calibration test. The box on the left includes a model of the data in the temperature range between 14 and $20^{\circ} \mathrm{C}$. After Luzi et al. (2021).

Figure 8 shows the time series of the displacement and temperature measured during the calibration. The strong correlation between these two variables is evident. Figure 9 shows a plot of displacement versus temperature. In this plot, there are two main regimes. The first one occurs with temperatures below $20{ }^{\circ} \mathrm{C}$ : in this range, the strongest dependence of displacement on temperature occurs. The linear model between displacement and temperature, shown in Figure 9 , has a $\mathrm{R}^{2}$ of 0.75 . The second regime occurs above $20^{\circ} \mathrm{C}$, showing a much weaker influence of temperature on displacement.

The linear behaviours shown in Figures 7 and 9 suggest the need of applying a correction to improve the accuracy of the retrieved phase, using the formula derived in the short-range calibration experiment. This was applied on the data plotted in Figure 6. Figure 10 shows the comparison between the original data and the data corrected using the equation shown in Figure 9. A significant improvement is achieved: the standard deviation of the time series drops from 3 to $1.6 \mathrm{~mm}$. This represents an encouraging result of the calibration, which is a valuable starting point to set up new improvements. The final goal is to achieve an error dispersion of the displacements below $1 \mathrm{~mm}$.

\section{CONCLUSIONS}

In this paper, the design, implementation, and testing of a lowcost, moderate performance AR have been described. The AR is designed to work in C-band with the data acquired by the Sentinel-1 sensors (operating at $5.405 \mathrm{GHz} \pm 50 \mathrm{MHz}$ ).

A brief description of the AR development has been provided. The AR consists of two antennas and an amplifying section, powered by a battery or any other available power source. The development of the device involved the design of a specific antenna (a 4 by 4 linear patch array). By contrast, the amplifying section was built using off-the-shelf components. It is worth mentioning that the production cost of the latest prototype is below 1000 euros. However, the planned integration of the radio frequency section could reduce the cost to a few hundred euros.

The AR has been tested in different experiments. In this paper, the experiment carried out in the PMT has been described. The device demonstrated satisfactory performances in terms of RCS, guaranteeing high visibility in an agricultural field, providing an adequate signal to noise ratio. The displacement time series of the AR, shown in Figure 6, was used to assess the performance of the AR. This time series concerns a location that is expected to be stable (i.e. with zero displacements) in the observed period. However, it shows a strong correlation with the temperature of the scene in correspondence to the image acquisition dates.

This behaviour has been investigated setting out a calibration experiment in a controlled environment. The tests showed that there is a strong linear dependence of the phase (and hence the displacement) on temperature. This experiment was used to derive an experimental calibration curve. Such a curve was then applied to the data from the PMT measurement. Using this simple correction improved the performance of the AR.

This is a promising experimental result. It is worth noting that, due to the characteristics of the experiment, the dispersion of the displacement values shown in Figure 9 represents a rather pessimist estimation of the phase noise (displacement noise) dispersion. In fact, the dispersion includes the other phase components, e.g. the differential residual topographic error and the differential atmospheric phase component. That is to say, the phase stability performance should be slightly better than the one shown in this paper.

The next step is to make an accurate and comprehensive laboratory test to characterize the phase response of the device, evaluating the need of further improvements, which could involve different hardware aspects: thermal, electromagnetic and mechanical aspects. A farther step will be the assessment of the calibration procedure in different environmental measurement conditions. 


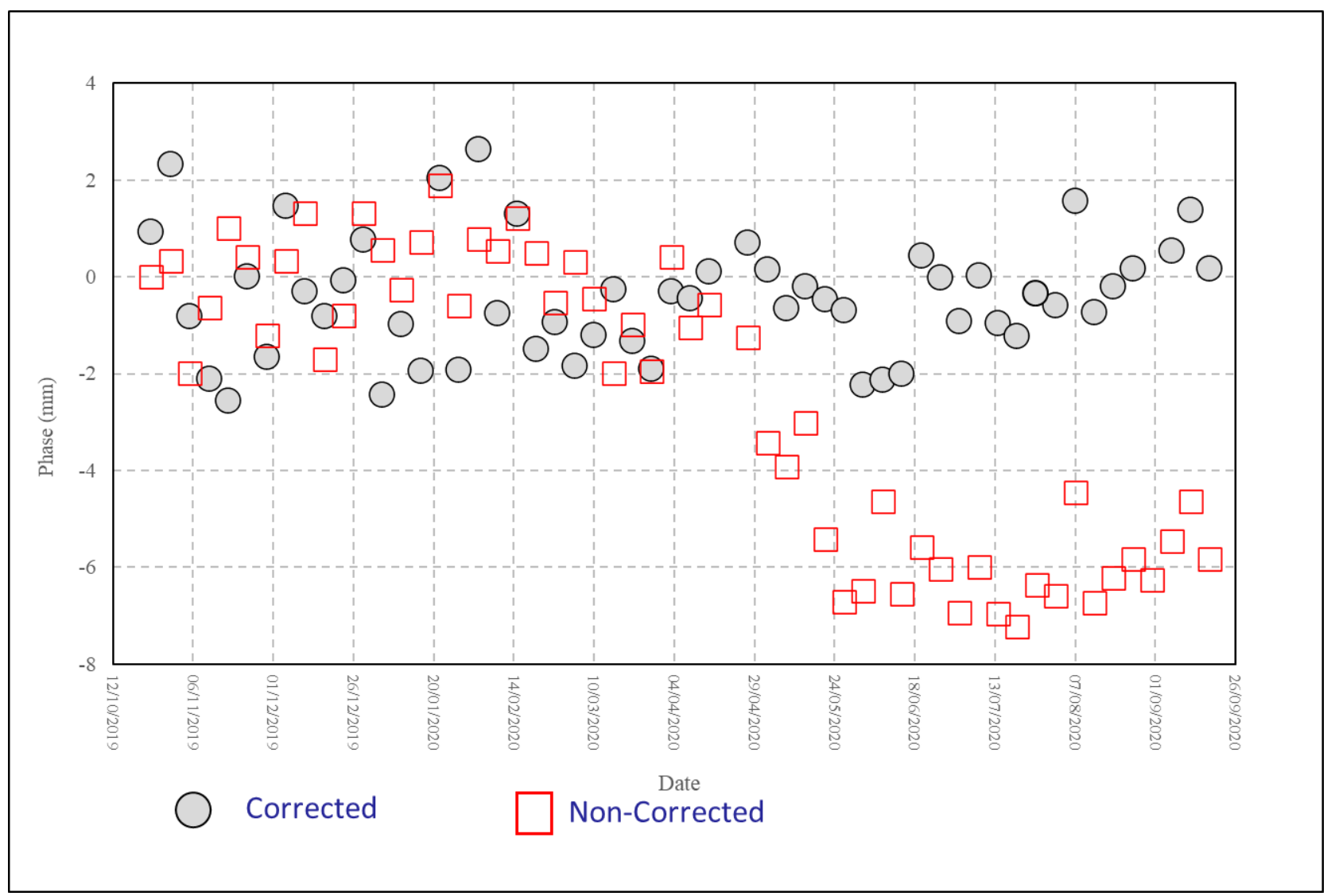

Figure 10. Cumulated deformation shown in Figure 6 (red squares) and corrected version using the equation shown in Figure 9 (circles). Modified from Luzi et al. (2021).

\section{ACKNOWLEDGEMENTS}

This work is part of a project that has received funding from the European GNSS Agency under the European Union's Horizon 2020 research and innovation programme, with grant agreement No 776335, project GIMS, "Geodetic Integrated Monitoring System". This work has been partially funded by AGAUR, Generalitat de Catalunya, through the Consolidated Research Group RSE, "Remote Sensing” (Ref: 2017-SGR-00729).

\section{REFERENCES}

Bamler, R., Hartl, P., 1998. Synthetic aperture radar interferometry. Inverse Probl, 14, R1-R54.

Crosetto, M., Gili, J.A., Monserrat, O., Cuevas-González, M., Corominas, J. and Serral, D., 2013. Interferometric SAR monitoring of the Vallcebre landslide (Spain) using corner reflectors. Nat. Hazards Earth Syst. Sci, 13(4), pp.923-933.

Crosetto, M., Monserrat, O., Cuevas-González, M. Devanthéry, N., Crippa, B., 2016. Persistent Scatterer Interferometry: a review. ISPRS Journal of Photogrammetry and Remote Sensing, 115, 78-89.

Freeman, A., Shen, Y. and Werner, C.L., 1990. Polarimetric SAR calibration experiment using active radar calibrators. IEEE Transactions on Geoscience and Remote Sensing, 28(2), pp.224-240.
Garthwaite, M., 2017. On the Design of Radar Corner Reflectors for Deformation Monitoring in Multi-Frequency InSAR. Remote Sensing, 9, 648.

Hanssen, R., 2001. Radar interferometry. Kluwer Academic Publishers, Dordrecht (The Netherlands).

Luzi, G., Espín-López, P.F., Mira Pérez, F., Monserrat, O. and Crosetto, M., 2021. A Low-Cost Active Reflector for Interferometric Monitoring Based on Sentinel-1 SAR Images. Sensors, 21(6), p. 2008.

Mahapatra, P.S., Samiei-Esfahany, S., van der Marel, H. and Hanssen, R.F., 2013. On the use of transponders as coherent radar targets for SAR interferometry. IEEE Transactions on geoscience and remote sensing, 52(3), pp.1869-1878.

Metasensing website, 2020. http://www.metasensing.com/docs /MetaSensing-ecr-c.pdf.

Rosen, P.A., Hensley, S., Joughin, I.R., Li, F.K., Madsen, S.N., Rodriguez, E., Goldstein, R.M., 2000. Synthetic aperture radar interferometry. Proceedings of the IEEE, 88(3), 333-382.

Skolnik, M., 1990. Radar Handbook. Mc Graw Hill Publishing Company: New York, NY, USA, 1990. 